\title{
Can NSAIDs contribute to Alzheimer's disease?
}

Q everal years ago it was dis$\checkmark$ covered that some NSAIDs lower the levels of amyloid $\beta$ peptide $(\mathrm{A} \beta)$. This peptide is a key component of neuritic plaques, which, along with neurofibrillary tangles and cerebral atrophy, are a pathologic hallmark of Alzheimer's disease. Not all NSAIDs produce this result, however, and new research reveals that some cyclooxygenase-2 (COX-2) inhibitors actually increase the production of $\mathrm{A} \beta$, an effect comparable to that of mutations thought to cause Alzheimer's disease. ${ }^{1}$

There are several forms of $\mathrm{A} \beta$; the one with 42 amino acids (A $\beta 42)$ is the insoluble form found in neuritic plaques. The deposition of $A \beta 42$ is thought to play an important role in the cascade of events leading to the formation of neurofibrillary tangles and the eventual degeneration of neurons. The production of $A \beta 42$ is catalyzed by $\beta$ - and $\gamma$ secretases. In 2001, Weggen and colleagues found that the NSAIDs ibuprofen, indomethacin and sulindac sulfide decrease the levels of $\mathrm{A} \beta 42$ in cells, in some cases by as much as $80 \%$. $^{2}$ This decrease appears not to be due to an effect on cyclooxygenase, the primary target of NSAIDs. Instead, these drugs appear to modulate the ability of $\gamma$-secretase to produce $A \beta 42$.

The results of a number of epidemiological studies that preceded the study by Weggen and colleagues suggested that people using NSAIDs had a reduced incidence of Alzheimer's disease. This led to speculation that NSAIDs may aid in preventing this disease. However, clinical trials with rofecoxib and naproxen showed no benefit. A prevention trial for Alzheimer's disease using celecoxib was recently halted because COX-2 inhibitors were coming under fire for increasing the risk of adverse cardiovascular events. ${ }^{3}$

\section{Mimicking Alzheimer's disease}

In a recent study, Kukar and colleagues tested over 300 compounds, including COX-2 selective NSAIDs, NSAID derivatives and several novel compounds, with the goal of finding drugs that had little effect on cyclooxygenase but decreased the production of A $\beta 42 .{ }^{1}$ They exposed cultured $\mathrm{H} 4$ neuroglioma cells to various drugs and measured production of $A \beta$.

Their results were surprising. Although they discovered that one NSAID, R-flurbiprofen, lacked cyclooxygenase activity and reduced $\mathrm{A} \beta 42$ levels, many of the COX-2 inhibitors, including tilmacoxib and valdecoxib, increased the production of $A \beta 42$. In particular, celecoxib raised $A \beta 42$ levels by almost $200 \%$. Rofecoxib and lumiracoxib only slightly influenced $\mathrm{A} \beta 42$ levels, even at high doses. The COX-2 selective NSAIDs appeared to exert their effects on $\mathrm{A} \beta 42$ production by targeting $\gamma$-secretase. This is similar to the mechanism by which autosomal dominant mutations linked to Alzheimer's disease are known to function.

NSAIDs are not the only class of drug that affects $A \beta 42$ production. Fenofibrate, a lipid-lowering drug, increased the $A \beta 42$ levels in cultured $\mathrm{H} 4$ cells by over $300 \%$ in a dose-dependent fashion. In contrast, fenofibric acid, the active metabolite of fenofibrate, did not raise $\mathrm{A} \beta 42$ levels, which suggests that the effect was specific to the drug.

The clinical implications of these findings are not clear, as the authors point out. ${ }^{1}$ In particular, it is uncertain whether celecoxib and the other drugs

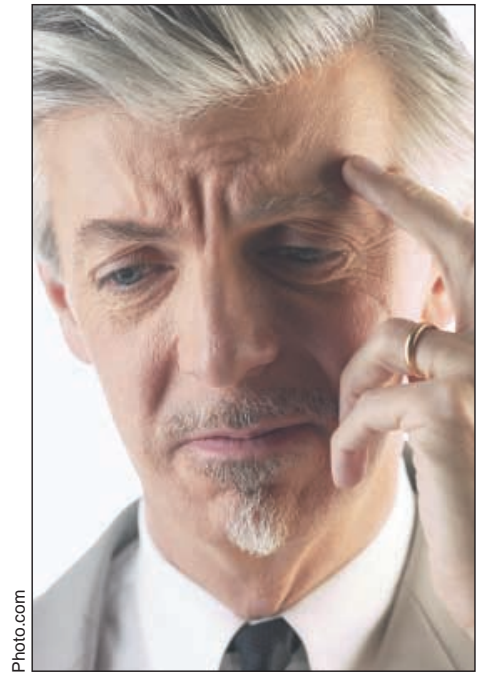

used in the study have the same effects in the human brain as they did in the mouse model used in the study. If they do, it is conceivable that they may have an impact on the pathology of Alzheimer's disease, which raises several issues for future study. The in vivo effects of COX-2 inhibitors on A $\beta 42$ levels and subsequent neuritic plaque formation need to be ascertained. An understanding of how these NSAIDs modulate the activity of $\gamma$-secretase may one day contribute to the design of drugs that inhibit the production of $A \beta .^{3}$ Finally, whether the drug-induced alteration of $\mathrm{A} \beta 42$ levels is a new risk factor for Alzheimer's disease needs further research. - David Secko, Vancouver

\section{References}

1. Kukar T, Murphy MP, Eriksen JL, Sagi SA, Weggen S, Smith TE, et al. Diverse compounds mimic Alzheimer disease-causing mutations by augmenting Abeta42 production. Nat Med 2005;11(6):545-50.

2. Weggen S, Eriksen JL, Das P, Sagi SA, Wang R, Pietrzik CU, et al. A subset of NSAIDs lower amyloidogenic Abeta42 independently of cyclooxygenase activity. Nature 2001; 414(6860):212-6.

3. Wyss-Coray T. Killing pain, killing neurons? Nat Med 2005;11(5):472-3. 